\title{
El "Wow factor". Experiencias de aprendizaje en la asignatura Taller I: Conservación y Restauración de Bienes Culturales
}

\section{Antoni Colomina Subiela ${ }^{a}$}

aUniversitat Politècnica de València. ancosu@upvnet.upv.es

\begin{abstract}
Different pedagogical currents confirm that surprise impulses beneficially affect in the learning processes, and also these incitations are enhanced in their effectiveness when they are accompanied by some dose of laughs or good humor. Both theorical explanations, developed through demostrative methods, and practical activities in the laboratory, are more efficient to the extent that the "wow factor" is included as a catalytic element.

In the last academic years it has been put into practice, in the subject "Laboratory I" in the degree in conservation and restoration of cultural assets, diverse surprise acts. The innovation has been carried out in line with a methodology of flipped classroom, obtaining satisfactory results that are linked to the establishment of a suggestive and attractive work environment for students in order to develop a meaningful learning.
\end{abstract}

Keywords: wow factor, gamification, TIC, laugh, good mood, conservation and restoration, flipped classroom, meaningful learning.

\section{Resumen}

Diferentes corrientes pedagógicas atestiguan que los estímulos sorpresivos redundan beneficiosamente en los procesos de aprendizaje y que, además, estas incitaciones ven acrecentada su eficacia cuando vienen acompañadas de alguna dosis de risa o buen humor. Tanto las explicaciones teóricas, desarrolladas a través de métodos expositivos, como las actividades prácticas en el entorno del laboratorio o taller, son tanto más eficientes en la medida en que el "wow factor" se incluye como elemento catalizador.

Durante los últimos cursos académicos se han implementado diferentes actos sorpresivos en la asignatura "Taller I", del Grado en Conservación y Restauración de Bienes Culturales de la Universitat Politècnica de València. La innovación se ha llevado a cabo en consonancia con una metodología de docencia inversa, obteniendo unos resultados satisfactorios que se vinculan al establecimiento de un entorno de trabajo sugestivo y atrayente para que el alumnado desarrolle un aprendizaje significativo. 
El "Wow factor". Experiencias de aprendizaje en la asignatura Taller I: Conservación y Restauración de Bienes Culturales

Palabras clave: factor sorpresa, "wow factor", ludificación, TIC, risa, buen humor, conservación y restauración, docencia inversa, aprendizaje significativo.

\section{Introducción}

Los actos sorpresivos generan, especialmente durante la infancia, estímulos muy potentes que redundan en un aprendizaje duradero. Esta capacidad de sorpresa parece adormilarse a partir de la adolescencia (Ibáñez, 2016), bien por el desarrollo madurativo del individuo o, sencillamente, por los cambios sustanciales que se producen en los métodos didácticos. Es obvio que con el crecimiento la facilidad para impresionarse frente a los nuevos conocimientos puede verse reducida, pero es posible predisponer al alumno para que cultive una sensibilidad renovada que le permita amplificar su asombro, a la vez que se implementan métodos que garanticen la asimilación perdurable.

Las transformaciones de síntesis proteica que se generan en el cerebro cuando se producen los procesos memorísticos y aprehensivos son más enérgicas en cuanto y tanto se corresponden con estímulos imprevisibles. El efecto sorpresa de los acontecimientos inesperados favorece, de este modo, la asimilación del hecho novedoso y de los detalles de su entorno que sirven para contextualizarlo, viabilizando enormemente la mejora del aprendizaje (Bravo, 2015).

\subsection{El "Wow factor" o los acontecimientos inesperados}

Las conexiones comunicativas que se producen en los diferentes ámbitos de la realidad social implican el desarrollo de estrategias que garanticen la aceptación e interiorización de los mensajes por parte del receptor. La eficacia de esta asimilación supone la ordenación por parte del emisor de habilidades efectivas de transmisión, de acuerdo con los canales, códigos y contextos que condicionan el acto de comunicación.

En el ámbito de la gestión comercial y económica, por ejemplo, las empresas desarrollan diferentes técnicas de marketing con la finalidad de atraer y fidelizar a sus clientes, ofreciendo soluciones atractivas a sus necesidades $y$, en definitiva, resolviendo sus problemas particulares. En la política, del mismo modo, las medidas propuestas por los dirigentes se exponen con el propósito de que tengan fuerza sobre aquellos a quienes van dirigidas (Canel, 1999: 18). Y en otros campos, como ocurre con los medios de comunicación de masas, las prácticas persuasivas provocan importantes impactos sociales y psicológicos (Bryant y Zillmann, 1996: 8).

En todas las circunstancias donde se precisa establecer impresiones positivas y duraderas es necesario acudir a probadas estrategias de comunicación, que procuren un entorno dispuesto para la presentación de ideas y para la sugestión del público con una imagen verosímil (Jones, 2008). En todos estos casos, el factor sorpresa o "Wow factor" se postula como una de las herramientas más destacables, capaz de despertar la curiosidad de los individuos para crear estímulos perdurables. 
Es posible extrapolar estas estrategias de comunicación al ámbito de la educación con la finalidad de otorgar experiencias dignas de memoria o memorables, acudiendo a la implementación de sucesos creativos y diferenciados (Amado, 2014). En el campo de la pedagogía, diferentes teorías asocian la eficacia del aprendizaje con lo sorpresivo o novedoso. El modelo propuesto por los profesores Robert Rescorla y Allen Wagner (1972) constituye el caso más acreditado, que teoriza sobre el concepto de sorpresa, en cuanto que procura la adquisición de conocimientos de manera perdurable teniendo en cuenta la discrepancia entre lo que se espera que suceda y lo que sucede en realidad. De este modo, los seres humanos tendemos a recordar con mayor fuerza aquellos acontecimientos inesperados.

\subsection{Y, además, una sonrisa}

Las acciones sorpresivas que se proponen en el desarrollo de la innovación mantienen, además, una importante carga de ingenio, donde lo ocurrente salpica ligeramente las actividades para potenciar el estímulo de lo inesperado. El humor y la risa son agentes beneficiosos para la salud física y psicológica; y su uso en el aula, como estrategia pedagógica, sirve para facilitar los procesos de aprendizaje (Madrid, 2015).

Desde el punto de vista de la psicología positiva se justifica la incitación controlada de la risa en clase, confiando en su capacidad para desarrollar las facultades cognitivas del alumno y favorecer la acción práctica. En contraposición, se ha demostrado que, a nivel fisiológico, el estrés y la actividad bajo presión provocan la liberación de adrenalina, cortisol y vasopresina en el cerebro, dificultando significativamente los procesos de aprendizaje (Jensen, 1998). En cambio, experiencias docentes más amenas aumentan los niveles de endorfina, dopamina, norepinefrina y serotonina, generando emociones positivas para el desarrollo formativo del alumno (Wolfe, 2001).

La risa propicia, en definitiva y en nuestro caso, la adquisición de destrezas y habilidades personales para hacer frente a las problemáticas particulares que se prevén en el ámbito de la conservación de bienes culturales. Estas emociones positivas, acaban transformando la actitud del estudiante para convertirlo en una persona más creativa, con un mayor conocimiento de las casuísticas reales, pertinaz frente a las dificultades y más integrado socialmente (Fredrickson, 2003).

En relación con esta última particularidad, esto es, el desarrollo del alumno como individuo social, es importante incidir en el efecto catalizador del humor y la risa, como agentes favorecedores de las relaciones humanas (Provine, 1996). Esta capacidad de sociabilización hace posible, al mismo tiempo, una inclinación por parte del alumno hacia el trato y la conexión interpersonal, no solo con el resto de los compañeros, sino también con el profesor, con lo que se favorece la proximidad en la docencia y la eliminación de barreras psicológicas (Pirowicz, 2011: 85). Se establecen entonces vínculos afectivos y empáticos, al mismo tiempo que se potencian los sentimientos de identidad y pertenencia al grupo.

Pero el humor y la risa, en forma de bromas y chistes inteligentes, comentarios ingeniosos o chascarrillos, además de "establecer una mejor relación con los estudiantes, reducir el estrés y la ansiedad, gestionar el conflicto, proporcionar una recompensa emocional que motive la 
El "Wow factor". Experiencias de aprendizaje en la asignatura Taller I: Conservación y Restauración de Bienes Culturales

participación y el estudio, y comunicar la materia más eficazmente" (Jáuregui y Fernández, 2009: 203), puede servir para aligerar la carga plúmbea de las clases teóricas impartidas mediante el tradicional método expositivo. Todos estos recursos pueden intercalarse como signos orales de puntuación para hacer más atractiva la lección magistral, conjugándose además con otros matices como la espontaneidad, la sencillez terminológica, la gesticulación, la utilización de circunloquios o la entonación.

\section{Objetivos}

Las emociones positivas, como las que se desprenden de las sensaciones de sorpresa, el juego y el humor, puede ampliar y construir los recursos personales perdurables del individuo, que utilizará en el futuro (Fredrickson, 2000). A continuación se enumeran los objetivos fundamentales que persigue la implementación de las acciones relacionadas con el factor sorpresa:

- Sugestionar al alumnado con la finalidad de captar su completa atención, recurriendo a acciones sorpresivas.

- Concretar actividades que sirvan como catalizador del proceso de aprendizaje significativo.

- Motivar a los alumnos e involucrarlos en las dinámicas de cooperación y en los procesos de aprendizaje independiente o autónomo.

- Impulsar actividades que fomenten la creatividad, con modelos de aplicación práctica que apelen a la diferenciación y originalidad.

- Crear experiencias de aprendizaje diversas bajo un entorno de trabajo atrayente y ameno.

\section{Desarrollo de la innovación}

Todos estos conceptos relacionados con las experiencias sorpresivas y la agudeza ingeniosa han sido implementados en algunas sesiones de la asignatura "Taller I", incluía en el Grado en Conservación y Restauración de Bienes Culturales de la Facultad de Bellas Artes, en la Universitat Politècnica de València.

La asignatura se imparte durante el primer cuatrimestre del tercer curso de la titulación y se conecta de manera muy directa con otras asignaturas que le preceden y suceden. Por un lado, recoge los conocimientos desarrollados en el segundo curso, en especial, los relacionados con las técnicas, materiales y procedimientos pictóricos y escultóricos; por otra parte, sirve como experiencia introductoria a los tratamientos básicos de conservación de algunas de las principales tipologías de obras artísticas. Su carácter genérico hace que atienda a las problemáticas de la pintura de caballete sobre lienzo y tabla, la pintura mural, la escultura en soporte pétreo y la cerámica arqueológica. "Taller I" establece un punto de inicio, a partir del cual el alumno especifica su itinerario formativo de acuerdo con sus preferencias entre los diferentes tipos de bienes culturales. Esta especialización posterior se 
formaliza a través de las asignaturas "Taller II" y "Taller III", impartidas respectivamente en el segundo cuatrimestre del tercer curso y en el primer cuatrimestre del cuarto curso de la titulación.

En concreto, la innovación que se plantea ha sido desarrollada durante el primer cuatrimestre de los cursos 2017-2018 y 2018-2019, particularmente en la sección teórica de la asignatura destinada al estudio anatómico de la pintura sobre tabla, sus causas de alteración y los tratamientos básicos para su conservación y restauración.

Aunque la asignatura "Taller I" es esencialmente práctica, se fundamenta en unos contenidos teóricos muy básicos que el alumno debe controlar y que le capacitarán para la identificación de los materiales constitutivos de los bienes culturales y la verificación de su comportamiento frente a los principales agentes de deterioro. Para presentar estos contenidos la exposición oral puede resultar un buen método, establecido de acuerdo con las características más habituales que definen la lección magistral. Sin embargo, conforme a las corrientes pedagógicas contemporáneas, el método expositivo puede incurrir en el peligroso escenario del aprendizaje pasivo, meramente memorístico y descontextualizado.

Para activar el proceso de aprendizaje se proponen una serie de acciones que, como recurso común, apelan al "Wow factor" como herramienta de dinamización. De este modo, lo inesperado adquiere el total protagonismo en detrimento de lo previsible, despierta la curiosidad, produce estímulos positivos en el aula y fomenta un impulso optimista y animado frente a la asignatura. El factor sorpresa alimenta el aprendizaje activo y significativo, donde el alumno participa de su propio aprendizaje y, como estudiante de educación superior aprende reflexionando, debatiendo y haciendo. Más allá de convertirse en receptáculo de la información que se le transmite, está implicado en el desarrollo de sus habilidades, involucrándose en niveles cognitivos superiores y participando en actividades de análisis, síntesis y discusión (Bonwell y Eison, 1991: 1-5).

Tabla 1. Desarrollo de experiencias sorpresivas en la asignatura "Taller I"

\begin{tabular}{lll}
\hline Momento experiencial & \multicolumn{1}{c}{ Tipo de experiencia } & Objetivos o propósito de la actividad \\
\hline $\begin{array}{l}\text { Al inicio del bloque } \\
\text { temático }\end{array}$ & Actividad ludificada & $\begin{array}{l}\text { Evaluar el nivel cognitivo del grupo en } \\
\text { los momentos iniciales }\end{array}$ \\
$\begin{array}{l}\text { Durante el desarrollo de } \\
\text { las exposiciones orales }\end{array}$ & $\begin{array}{l}\text { Interpelación socrática } \\
\text { divertida }\end{array}$ & $\begin{array}{l}\text { Invitar a la reflexión personal y al debate } \\
\text { participativo durante las explicaciones } \\
\text { teóricas }\end{array}$ \\
En las prácticas de aula & $\begin{array}{l}\text { Actividades de trabajo en } \\
\text { equipo sobre supuestos } \\
\text { prácticos }\end{array}$ & $\begin{array}{l}\text { Ensayar casuísticas reales donde se } \\
\text { ponga a prueba la aplicación del } \\
\text { pensamiento crítico }\end{array}$ \\
$\begin{array}{l}\text { Alerta motivadora } \\
\text { Durante el trabajo }\end{array}$ & $\begin{array}{l}\text { Crear estímulos a través de las TIC para } \\
\text { animar al alumno en la realización de } \\
\text { sus tareas fuera del aula }\end{array}$ \\
\hline
\end{tabular}


El "Wow factor". Experiencias de aprendizaje en la asignatura Taller I: Conservación y Restauración de Bienes Culturales

A continuación y de acuerdo con la tabla 1, se exponen diferentes estrategias sorpresivas para dinamizar la transmisión de los contenidos teóricos de la asignatura y que se desarrollan en diferentes momentos del itinerario formativo: una actividad ludificada en el momento inicial de "Taller I"; acciones de debate reflexivo para complementar las explicaciones teóricas; prácticas de aula que implican trabajo cooperativo en equipo; y estímulos inesperados en el transcurso de las tareas fuera del aula, incluidas en dinámicas de docencia inversa.

\subsection{La evaluación inicial a través de la ludificación}

Para los momentos iniciales del bloque temático referido a la conservación de pintura sobre tabla se propone la realización de una actividad ludificada, para lo cual se recurrirá a la aplicación KAHOOT!. Se trata de un juego participativo de preguntas y respuestas tipo test, tras el cual se establece un ranking de acuerdo con los puntos obtenidos por cada participante, conforme al número de respuestas acertadas y la velocidad de acierto.

Las preguntas del concurso se proyectarán en clase y cada alumno, después de identificarse en el sistema, utilizará su dispositivo móvil como pulsador para seleccionar la respuesta que crea correcta. En este caso el juego constará de 20 cuestiones, a las que se les asignará un tiempo de respuesta de 20 segundos por pregunta.

De manera previa, de acuerdo con una metodología de clase inversa, el alumno necesitará haber revisado los apuntes de la asignatura "Materiales, técnicas y procedimientos pictóricos", de segundo curso de Grado, especialmente los referidos a la evolución histórica de la madera como soporte pictórico. El repaso general del tema propuesto implicará la actualización de las notas y apuntes del curso precedente. En este caso, la valoración del proceso de estudio se conectará con el resultado que se obtenga en la actividad ludificada programada para la sesión de clase presencial. 


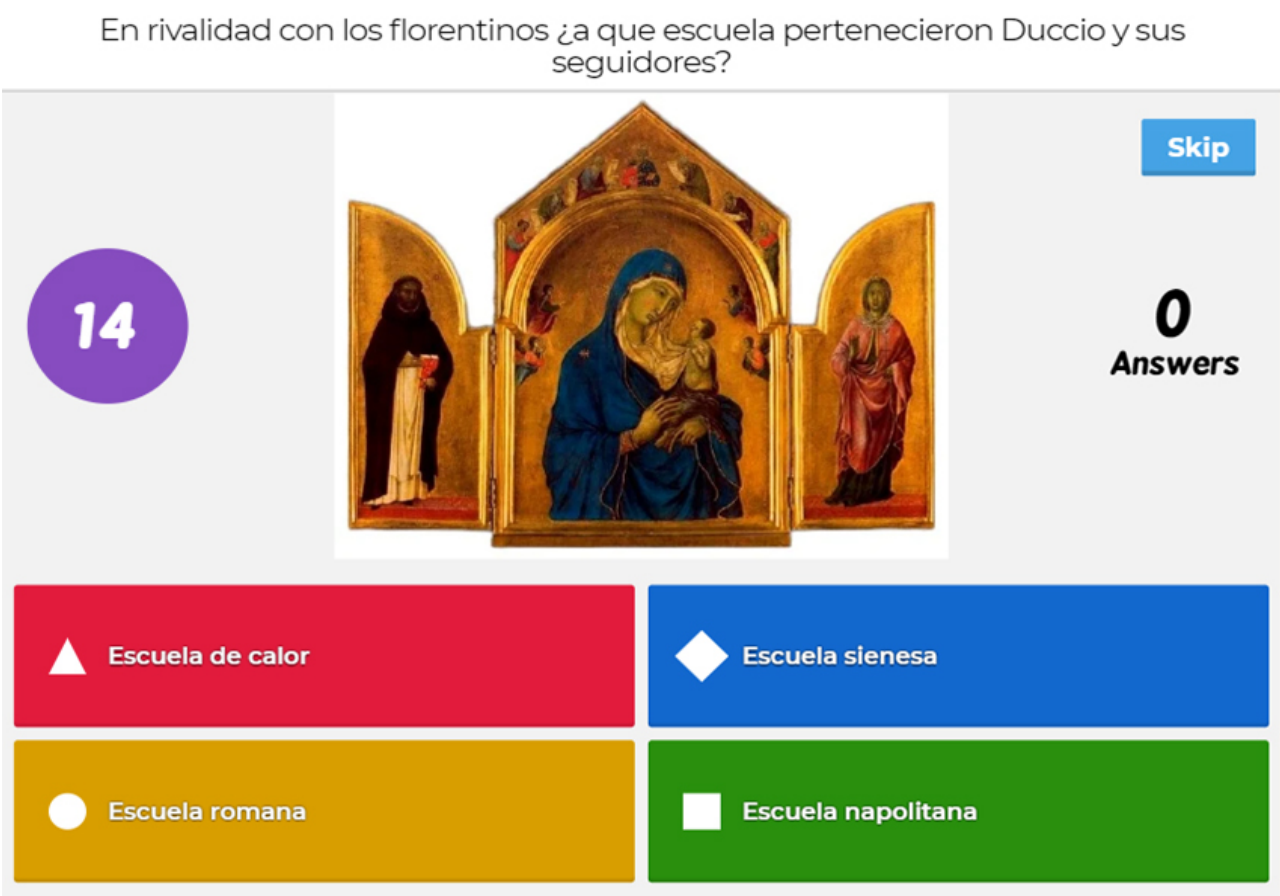

Fig. 1. Pregunta de la aplicación KAHOOT! que incluye, como opción roja, una respuesta errónea de tipo ingenioso, imposible y que incita a la hilaridad.

Los alumnos se erigen como verdaderos protagonistas de la sesión educativa, de acuerdo con una actividad de gran impacto emocional y que puede, además, estar salpicada de notas humorísticas. Entre las posibles respuestas, es muy eficiente incluir alguna opción imposible, desconcertante o ingeniosa que provoque momentos de complicidad e hilaridad, tal y como ocurre con la opción de color rojo de la figura 1.

Después de cada pregunta y según el número de aciertos, el profesor intervendrá para realizar alguna puntualización, aclarar dudas o reforzar alguno de los conceptos más básicos. La aplicación permite exportar los resultados a una tabla de Excel, de modo que será posible establecer una evaluación inicial del nivel cognitivo del grupo, para avanzar en la asignatura y, si es necesario, adecuar el resto de las actividades programadas para alcanzar con garantía los objetivos establecidos.

\subsection{La interpelación socrática}

La exposición oral o clase magistral, conforme al método expositivo tradicional, obedece a menudo a la declaración de conceptos que, según la condición del profesor, se convierten en dogmas irrebatibles. Es entonces cuando el proceso de aprendizaje se convierte en una mera transmisión de axiomas que solo merecen la retención memorística y la aceptación incuestionable.

Sin embargo, es posible apelar al método expositivo conforme a una actitud docente alternativa, donde los conocimientos expuestos por el profesor sean relativos y den cabida al debate, a las preguntas y a las críticas cognoscitivas (Charaja, 2014: 60). De este modo, 
El "Wow factor". Experiencias de aprendizaje en la asignatura Taller I: Conservación y Restauración de Bienes Culturales

la lección enraizada en una la actitud pasiva del alumno se convierte en una clase magistral interactiva (Morell, 2004), donde se establece una comunicación múltiple o bidireccional y la exposición es participativa.

Los planteamientos del profesor se presentan bajo la condición de que no responden necesariamente a una verdad absoluta $\mathrm{y}$, aunque se sustentan sólidamente en fuentes contrastadas, siempre están abiertos a nuevas revisiones. Se establece entonces una negociación epistémica entre los asistentes.

Estas clases teóricas interactivas quedan dinamizadas en la medida en que el profesor motive a la participación a través de la interpelación socrática, con la proyección de cuestiones para la reflexión y la discusión. Resulta eficaz plantear casos hipotéticos con preguntas directas que, en momentos puntuales, pillen a los alumnos desprevenidos o por sorpresa. Es habitual, al principio, la generación de un primer desconcierto que vendrá acompañado de respuestas incompletas (Díaz, 2015), pero que acabará por alertar al grupo y lo predispondrá a adoptar una actitud más vigilante.

La clase se dinamiza bajo un ambiente participativo e integrador. Lo sorpresivo quedará suspendido en la atmósfera y se reforzará, incluso, con algún cometario o representación cómica. Para ejemplificar esta circunstancia puede valer la figura 2, donde se reflexiona profundamente acerca de una frase o cita cinematográfica que, finalmente, se desvela su procedencia como comentario extraído de una popular película infantil.

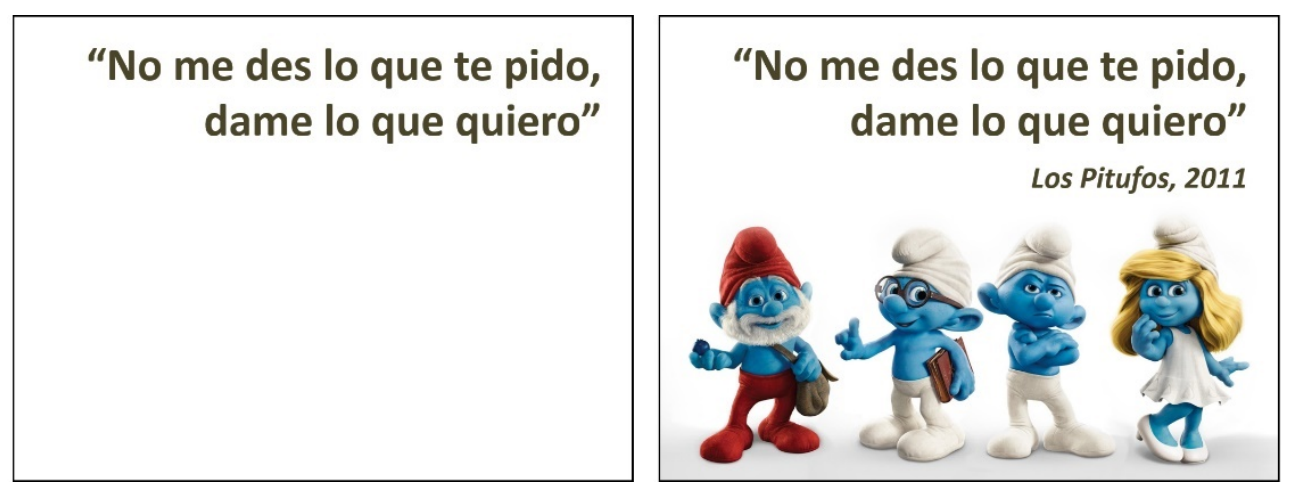

Fig. 2. Cita cinematográfica para la reflexión participativa.

\subsection{Prácticas de aula}

La aplicación de los contenidos teóricos de la asignatura encuentra en las prácticas de aula un entorno adecuado para su desarrollo. Se trata de actividades que, especialmente a través del trabajo cooperativo en equipo, resuelven algún tipo de planteamiento ficticio, en este caso, estrechamente vinculado con la conservación de la pintura sobre tabla. Para ello, el aula se convierte simuladamente en un taller profesional, donde suceden casos supuestos que se vinculan con la realidad del oficio.

Siguiendo la línea metodológica que hace uso del factor sorpresa se plantean dos actividades que pretenden generar expectación y despertar la curiosidad del alumnado 
desde sus momentos iniciales. Igualmente, esta provocación genera un ambiente de trabajo distendido y animado, que se reforzará con la inclusión de pequeñas anécdotas ocurrentes.

\subsubsection{La caja sorpresa}

Esta actividad se presenta como un supuesto próximo a la realidad profesional de un taller de conservación y restauración de bienes culturales. Su desarrollo se propone como conclusión de las clases teóricas de la asignatura, donde el alumnado, por equipos, deberá volcar lo aprendido en clase, utilizando la terminología técnica y aplicando el pensamiento crítico y relacional.

Sobre la mesa de cada grupo de trabajo se dispone una caja de cartón, cerrada, con la que los alumnos coincidirán en su llegada a clase. Este primer encuentro les hace mantenerse alerta y despierta su curiosidad. Se convierte, entonces, en un elemento desconcertante y que les mantiene expectantes durante los momentos iniciales de la sesión. Esta expectación se mantiene vigente en cuanto y tanto el profesor no desvela demasiados detalles de su contenido. Obviando su presencia se inician unas primeras explicaciones, que compendiarán los contenidos teóricos expuestos en las clases anteriores. La paciencia de los alumnos queda constatada, aunque se advierte algún intento tímido que pretende descubrir, sin éxito, el asunto del contenido.

Una vez revisados los conceptos más significativos de la sección, referidos a la restauración de la pintura sobre tabla, se invitará a cada grupo a levantar la tapa de sus respectivas cajas. En su interior aparecerán cinco miniaturas en cartón-pluma que representarán a escala diferentes pinturas renacentistas del artista Cristóbal Llorens, reproducidas tanto por su anverso como por su reverso conforme a la figura 3 . 


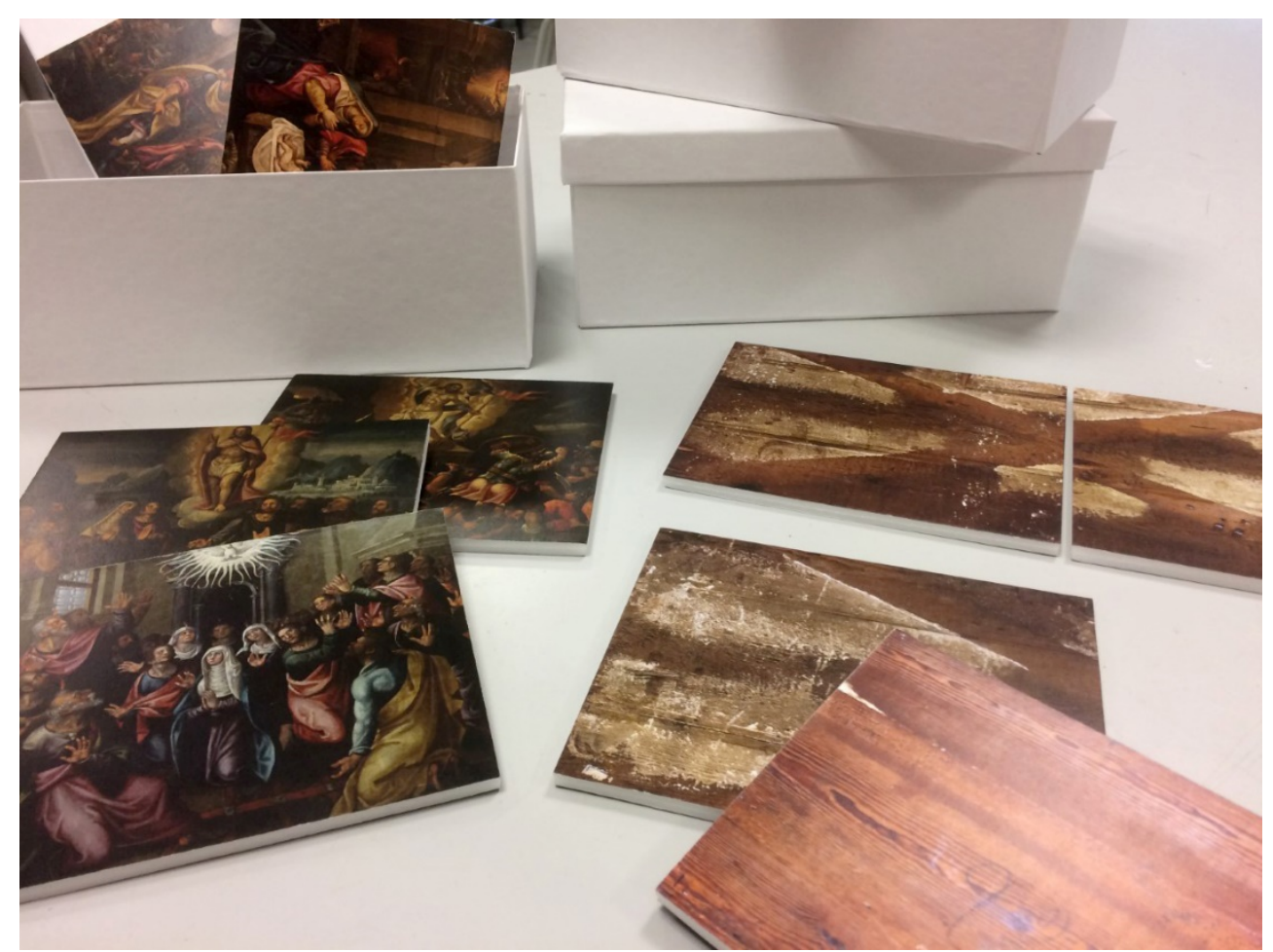

Fig. 3. Reproducción a escala de diferentes pinturas sobre tabla del artista Cristóbal Llorens.

Los equipos de trabajo deberán cumplimentar unas fichas donde se hará constar la iconografía de cada obra, sus peculiaridades técnicas y su estado de conservación. Además, con todo lo explicado en clase, deberán ser capaces de concluir que todas las piezas formaron parte de la predela de un mismo retablo y que existe entre ellas una correspondencia muy próxima.

Todos sus análisis, finalmente, quedarán expuestos en público a través de un portavoz de equipo, que presentará frente al resto de compañeros las averiguaciones referidas a cada tabla en particular y a la conexión que existe entre todo el conjunto de obras.

\subsubsection{La llamada misteriosa}

Del mismo modo que sucede con la actividad anterior, esta segunda propuesta desarrolla un suceso fingido que, perfectamente, podría darse en el contexto de trabajo de un taller de restauración institucional. Por tratarse de un caso próximo a la realidad del oficio, incluye factores incontrolados que, por lo habitual, no suelen contemplarse durante el periodo formativo del alumno.

La actividad comienza con la explicación teatralizada del profesor, que debe excusar su ausencia durante unos minutos, de modo que abandona el aula para visitar, supuestamente, un retablo pictórico de una iglesia próxima. Realiza una llamada telefónica al representante de la clase que, según las indicaciones que reciba, deberá transmitir a sus compañeros las características estructurales de la obra frente a la que se encuentra el profesor. En su descripción, utilizará la terminología técnica aprendida en las sesiones teóricas precedentes, 
a través de la cual los alumnos deberán trazar un croquis de la obra, con su estructura y dimensiones, según se observa en la figura 4.
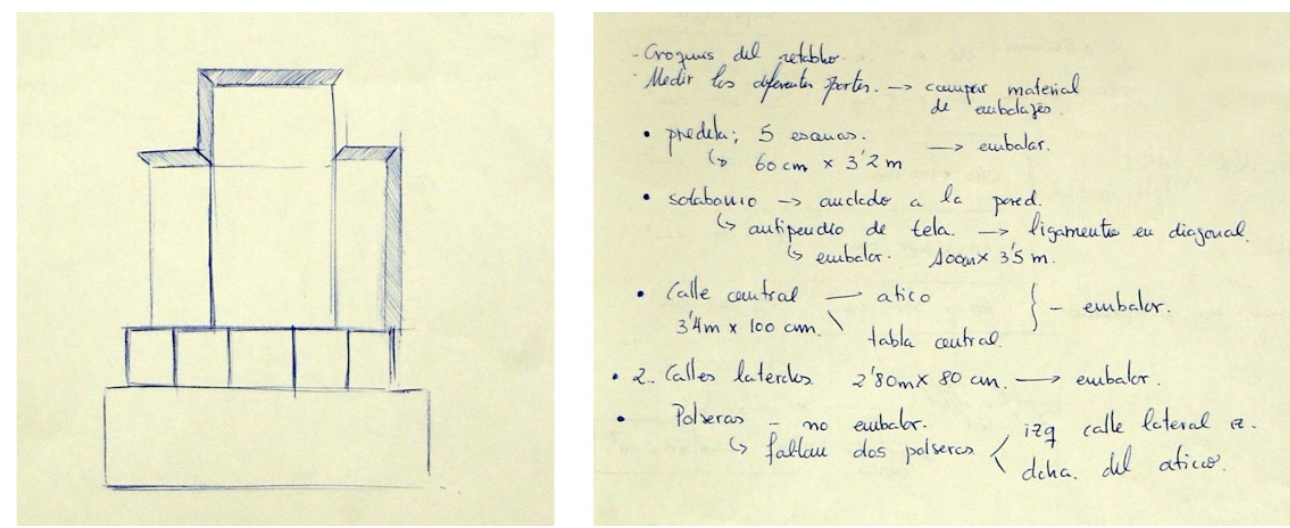

Fig. 4. Esbozo de la estructura de un retablo y notas sobre sus elementos compositivos y dimensiones.

Finalmente, de acuerdo con las particularidades del retablo y el tamaño de cada una de las piezas que lo componen, deberán establecerse la cantidad total de material de embalaje que se necesitaría para transportarlo al taller para su intervención.

Con el retorno del profesor al aula se revisarán nuevamente todos los detalles de la experiencia. En la pizarra, junto con la participación de los alumnos, se trazará la estructura constructiva de la obra y se repasarán los cálculos para su manipulación.

\subsection{La estimulación sorpresiva en la docencia inversa}

Con la finalidad de ensayar los métodos pedagógicos actuales, muy acordes con la carga de trabajo que debe asumir el alumno según el sistema europeo de transferencia y acumulación de créditos (ECTS), establecidos con el Plan de Bolonia, la asignatura contempla la docencia inversa. El flip teaching se establece para algunos tramos de la asignatura "Taller I", en concreto para impartir los contenidos teóricos de la sección de conservación de pintura sobre tabla.

A través de la herramienta Lessons de la plataforma PoliformaT, intranet docente de la UPV, se ordena una guía de trabajo donde se especifican las actividades que, de acuerdo con la docencia inversa, el alumno debe realizar. Se detallan los diferentes trayectos que se relacionan con cada unidad temática y se determinan los ejercicios no presenciales que tienen que cumplirse, tanto de manera previa como con posterioridad a cada sesión teórica en clase.

Es muy difícil por parte del profesor llevar a cabo un seguimiento en tiempo real de las tareas encomendadas como trabajo autónomo, pues, precisamente por su carácter independiente, su ejecución queda supeditada íntegramente a la voluntad y predisposición de cada alumno. Sin embargo, es posible remitir ciertas alertas que ayuden a estimularlo e inciten a la acción. Según esto se proponen algunas dinámicas muy simples que, de igual 
El "Wow factor". Experiencias de aprendizaje en la asignatura Taller I: Conservación y Restauración de Bienes Culturales

manera, se cimentan en la idea del factor sorpresa, en este caso, con el objetivo de animar al estudio individual.

\subsubsection{La motivación a través del recurso musical}

En los momentos en los que el alumno debe responsabilizarse de la consecución de alguna tarea fuera del aula, es posible que tropiece con algunos obstáculos que se deriven de la apatía o el agotamiento. Esta falta de actitud puede encontrar en la emoción un recurso esencial, capaz de revertir la indiferencia y la falta de dedicación para promover el aprendizaje (Albornoz, 2009).

La música puede servir como catalizador de emociones positivas, que inviten a la introspección personal y desarrollen las habilidades individuales que son necesarias para completar los procesos cognitivos. En la innovación que nos ocupa, en concreto, se hace uso del clip musical de la canción "Can't stop the feeling!", del artista estadounidense Justin Timberlake. La visualización del videoclip aparece integrada como actividad en el guion de trabajo establecido en Lessons. El carácter pop de la canción, como referente cercano y actual, la conectan de manera íntima con el estudiante, que se sentirá identificado con su contenido visual y mensaje inclusivo.

El alumno deberá prestar atención al significado general del tema y a su letra, especialmente a las líneas "I got that sunshine in my pocket / Got that good soul in my feet". Estas palabras propiciarán un acto de reflexión que ayudará a afrontar los trabajos propuestos con curiosidad y actitud positiva, en cuanto y tanto, cada individuo sea consciente de sus fortalezas y las aproveche para la consecución de los resultados de aprendizaje que se le exigen.

\subsubsection{La encuesta Doodle}

Como materiales complementarios para las actividades de docencia inversa se establecieron encuestas on-line para motivar el trabajo en casa. Así, durante el transcurso de las tareas encomendadas al alumno para que complete de manera no presencial el aprendizaje de cada unidad didáctica se recurre a remitir pequeños desafíos. A través de un correo electrónico que exponga un problema específico cercano a un caso real, como el mostrado en la figura 5 , se invita a participar en una encuesta Doodle, donde es posible seleccionar entre varias opciones la solución que se considere más oportuna para afrontar la cuestión planteada. Esto ayudará al estudiante a revisar los conceptos básicos para concluir el trayecto de formación, a la vez que servirá de estímulo. 


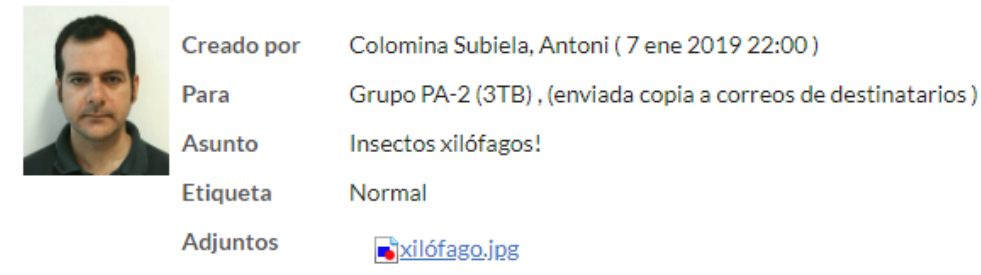

Holi!

He estado revisando vuestros trabajos esta mañana después de clase y parece que tenemos en el taller algún "visitante" inesperado. Se trata de algún tipo de insecto xilófago que se encuentra activo en alguna de las tablas.

No os alarméis, procederemos rápidamente a su desinsectación. No obstante, antes deberíamos saber con qué especie estamos tratando. ¿Nos podríais ayudar?

No hemos localizado ningún imago, pero su orificio de salida es redondo, de unos 2-3 mm de diámetro. Junto a estos orificios aparece un polvo o serrín bastante granuloso. Os adjunto una fotografía del caso en cuestión.

Podéis indicar, a lo largo de los próximos días, qué tipo de insecto se ha colado en clase a través de la siguiente encuesta: https://doodle.com/poll/cs6ecswwmazrraip

Gracias! :)

Toni

Fig. 5. Correo electrónico para la participación en encuestas Doodle.

\section{Resultados}

Sería necesario establecer un estudio profundo que estimara la repercusión real del factor sorpresa como recurso didáctico, con una evaluación pedagógica que contemplara las variables que entran en juego en los procesos de aprendizaje. Por el momento, la valoración de este tipo de innovación se establece, por un lado, considerando los resultados satisfactorios de las encuestas de alumnado. De acuerdo con la estimación efectuada por los alumnos a través de este sistema de control de la calidad docente se concluye que la satisfacción general ha sido óptima, con una media de 9,54 sobre 10 en este tramo de la asignatura. Entre los ítems que merecen especial atención y que se relacionan con la innovación se encuentran:

- La metodología empleada y las actividades realizadas en la asignatura ayudan a aprender al alumnado $(9,08)$.

- El profesor ontribuye a crear un buen clima de trabajo y anima a los alumnos a participar en las clases $(9,75)$.

- Lu manera de explicar del profesor consigue motivar al alumnado y despertar el interés por la asignatura $(9,33)$. 
El "Wow factor". Experiencias de aprendizaje en la asignatura Taller I: Conservación y Restauración de Bienes Culturales

La innovación docente, al estar incluida además en un programa de docencia inversa, ha sido evaluada igualmente a través de una segunda batería de encuestas. En este caso, y aunque los estudiantes detectan un esfuerzo considerable en el momento de desarrollar actividades fuera del aula, coinciden finalmente en los efectos positivos del método. En última instancia, esta carga de trabajo es asumida con ligereza en cuanto y tanto se admite y vincula con estímulos y actos sorpresivos. Algunos de los puntos destacables de este cuestionario evaluativo, estimados positivamente por el alumno, son:

- Aumento de la satisfacción del alumno.

- Fomento del trabajo en grupo.

- Aprendizaje más activo y experiencial.

- Interacción con profesor y compañeros más frecuente y positiva.

Por otra parte, los actos de evaluación sumativa para establecer balances fiables de los logros obtenidos al final del proceso de aprendizaje, tanto en lo referente a las pruebas teóricas de tipo escrito como a las experiencias prácticas, han revelado la correcta asimilación de los contenidos de la asignatura por parte del alumnado. En este sentido, se estima que los objetivos planteados de inicio se han alcanzado con éxito a nivel general, a la vez que se han desarrollado las destrezas y habilidades propias de las competencias transversales relacionadas con las peculiaridades de "Taller I".

\section{Conclusiones}

En definitiva, las experiencias de enseñanza basadas en el factor sorpresa pretenden mantener un nivel de expectación constante durante todo el transcurso del proceso de aprendizaje, previniendo la pérdida de interés por la asignatura $\mathrm{y}$, en consecuencia, la inactividad o pasividad por parte del alumno.

El "Wow factor" se vincula de manera muy directa con la risa y el buen humor en el aula, lo que fomenta, además, un entorno de trabajo cómodo y sugestivo. Un buen control de estos estímulos positivos proporciona un contexto idóneo, tanto para el trabajo cooperativo como para alentar al alumno en los procesos de estudio autónomo o autoaprendizaje. Se trata, en cierto modo, de buscar códigos reconocibles que favorezcan los vínculos de pertenencia al grupo de trabajo, con canales de comunicación con los que los alumnos se sientan identificados y con un lenguaje próximo y acorde con su particularidad generacional.

\section{Referencias}

ALBORNOZ, Y. (2009). "Emoción, música y aprendizaje significativo". Educere, 13 (44), pp. 67-73. $\quad<$ http://www.scielo.org.ve/scielo.php?script=sci_arttext\&pid=S131649102009000100008\&lng=es\&tlng=es $>$ [Consulta: 13 de enero de 2019]. 
AMADO, F. (2014). "El 'Wow factor' o factor sorpresa en la educación del IE" en $I E$ Claustro, 17 de febrero. <https://vdcav.blogs.ie.edu/2014/02/17/el-wow-factor-o-factorsorpresa-en-la-educacion-del-ie/> [Consulta: 31 de diciembre de 2018].

BONWELL, C. C. and EISON, J. A. (1991). Active learning; creating excitement in the classroom. ASHE-ERIC Higher Education Report No. 1. Washington, D.C.: The George Washington University, School of Education and Human Development.

BRAVO, M. G. (2015). “¿Funciona el efecto sorpresa sobre el aprendizaje?” en Iberoamérica divulga, 24 de octubre. $<$ https://www.oei.es/historico/divulgacioncientifica/?Funciona-el-efecto-sorpresa-sobre> [Consulta: 01 de enero de 2019].

BRYANT, J. y ZILLMANN, D. (coord.) (1996). Los efectos de los medios de comunicación: investigaciones y teorías. Barcelona: Paidós Ibérica.

CANEL, M. J. (1999). Comunicación política. Una guía para su estudio y práctica. Madrid: Tecnos.

CHARAJA, F. (2014). "Vigencia de la clase magistral en la universidad del siglo XXI". Apuntes Universitarios. Revista de Investigación, 4 (1), pp. 57-66. $<$ http://www.redalyc.org/articulo.oa?id=467646128005> [Consulta: 06 de enero de 2019].

DÍAZ, S. A. (2015). "El factor sorpresa puede crear como efecto colateral un aprendizaje significativo y además una sonrisa" en Iberoamérica divulga, 31 de octubre. $<$ https://www.oei.es/historico/divulgacioncientifica/?El-factor-sorpresa-puede-puede> [Consulta: 06 de enero de 2019].

FREDRICKSON, B. (2003). "The value of positive emotions". American Scientist, 91, $330-$

335.<https://www.americanscientist.org/sites/americanscientist.org/files/20058214332_306 .pdf> [Consulta: 02 de enero de 2019].

FREDRICKSON, B. (2000). "Cultivating positive emotions to optimize health and wellbeing”. Prevention \& Treatment, 3 (1), article ID 1.

IBÁÑEZ, A. (2016). "El efecto sorpresa en el aprendizaje" en Cerebro Adolescente, 25 de marzo. $<$ https://aranzazu5.blogspot.com/2014/07/el-efecto-sorpresa-en-el-aprendizaje.html $>$ [Consulta: 30 de diciembre de 2018].

JÁUREGUI, E. y FERNÁNDEZ, J. D. (2009). "Risa y aprendizaje: el papel del humor en la labor docente". Revista Interuniversitaria de Formación del Profesorado, 66 (23,3), pp. 203-215. < < <ttps://www.aufop.com/aufop/uploaded_files/articulos/1258588621.pdf> [Consulta: 04 de diciembre de 2019].

JENSEN, E. (1998). "How Julie's brain learns". Educational Leadership. How the Brain Learns, 56 (3), pp. 41-45.

JONES, F. C. (2008). How to wow: proven strategies for presenting your ideas, persuading your audience, and perfecting your image. New York: Ballantine Books. 
El "Wow factor". Experiencias de aprendizaje en la asignatura Taller I: Conservación y Restauración de Bienes Culturales

MADRID, J. (2015). "Papel de la risa y el humor en la enseñanza y aprendizaje: explicaciones neurofisiológicas". Apuntes Universitarios. Revista de Investigación [en línea], 5 (2), pp. 41-55. <http://www.redalyc.org/articulo.oa?id=467646280003> [Consulta: 02 de enero de 2019].

MORELL, T. (2004). La interacción en la clase magistral. San Vicente del Raspeig: Universidad de Alicante.

PIROWICZ, D. (2011). El humor en los procesos de enseñanza-aprendizaje. Tesis de Maestría. Buenos Aires: FLACSO, Sede Académica Argentina. $<$ http://repositorio.flacsoandes.edu.ec/bitstream/10469/3381/3/TFLACSO-01-2011DP.pdf $>$ [Consulta: 04 de enero de 2019].

PROVINE, R. (1996). "Laughter". American Scientist, 84 (1), pp. 38-47. $<$ http://cogweb.ucla.edu/Abstracts/Provine_96.html> [Consulta: 04 de enero de 2019].

RESCORLA, R. A. and WAGNER, A. R. (1972). "A theory of Pavlovian conditioning: variations in the effectiveness of reinforcement and non reinforcement" in A.H. Black, A. H. and Prokasy, W.F. (eds.), Classical conditioning II: current research and theory. New York: Appleton-Century-Crofts. pp. 64-99.

WOLFE, P. (2001). Brain Matters: Translating research into classroom practice. Virginia, USA: ASCD. 